\title{
From Routine to Research Laboratory: Strategies for the Diagnosis of Congenital Fibrinogen Disorders
}

\author{
Alessandro Casini ${ }^{1}$ \\ ${ }^{1}$ Division of Angiology and Hemostasis, University Hospitals of \\ Geneva, Geneva, Switzerland \\ Hämostaseologie 2020;40:460-466.
}

\begin{abstract}
Address for correspondence Alessandro Casini, MD, Division of Angiology and Hemostasis, University Hospitals of Geneva, Rue Gabrielle-Perret-Gentil 4, 1201 Geneva, Switzerland (e-mail: Alessandro.casini@hcuge.ch).
\end{abstract}

\begin{abstract}
Keywords

- fibrinogen

- fibrin

- coagulation factors

- mutations

- diagnosis

Congenital fibrinogen disorders (CFDs) encompass a heterogeneous group of fibrinogen defects with a wide spectrum of biological and clinical features. An accurate diagnosis is thus essential to assure the optimal management for the patient. Diagnosis involves a multistep approach starting with routine coagulation assays and assessment of functional and antigenic fibrinogen followed by identification of the molecular anomaly. However, the diagnosis of CFD can be challenging as the sensitivity and specificity of coagulation assays depend on the fibrinogen level as well as on the fibrinogen variant. In addition, patients suffering from CFD have a heterogeneous clinical course which is often unpredictable by routine coagulation assays. To better determine the patient's clinical phenotype, global hemostasis assays and an assessment of the fibrin clot properties are performed in research laboratories. In this review, we summarize the fibrinogen work-up highlighting some common pitfalls and provide an update of the research on CFD.
\end{abstract}

\section{Introduction}

Fibrinogen is a complex protein with multiple physiologic roles. ${ }^{1}$ Each fibrinogen molecule consists of two identical subunits, formed by three chains termed $A \alpha, B \beta$, and $\gamma^{2}$ The fibrinogen structure comprises two terminal $D$ regions both with seven structural domains and one central $\mathrm{E}$ region with four structural domains. ${ }^{3}$ The complexity of fibrinogen molecule is reflected by the wide biological and clinical spectrum of congenital fibrinogen disorders (CFDs).

CFDs are characterized by deficiency and/or dysfunctional fibrinogen resulting from monoallelic or biallelic mutations in FGA, FGB, and FGG genes on chromosome $4 .{ }^{4}$ Classically, CFDs have been classified into quantitative fibrinogen disorders (afibrinogenemia and hypofibrinogenemia) or qualitative fibrinogen disorders (dysfibrinogenemia and hypodysfibrinogenemia). ${ }^{5}$ Prevalence of afibrinogenemia is estimated to be around $1: 1,000,000$ of persons but is more common in countries where consanguineous marriages are common. A systematic analysis of exome/genome data from approximately 140,000

received

April 23, 2020

accepted after revision

May 19, 2020

individuals belonging to the genome Aggregation Database estimated that hypofibrinogenemia or dysfibrinogenemia represents a far more frequent condition in the population than initially thought. ${ }^{6}$ Recently, the Factor XIII and Fibrinogen SSC Subcommittee of the International Society of Thrombosis and Haemostasis (ISTH) has updated the classification of CFD taking into account the fibrinogen levels, the genotype, and the clinical phenotype of the patients. Several types and subtypes of fibrinogen disorders have been identified, each with specific biological and clinical features ${ }^{7}$ ( - Table 1 ). The main symptom of patients with quantitative CFD is bleeding, which is strongly dependent on the fibrinogen level. Indeed, fibrinogen levels $>0.7 \mathrm{~g} / \mathrm{L}$ seems to protect from spontaneous bleeding. ${ }^{8}$ Bleeding is the principal symptom of afibrinogenemia, but the severity is broad ranging from recurrent life-threatening cerebral bleeding to minor occasional bleeding. ${ }^{9}$ Furthermore, patients with afibrinogenemia are paradoxically at increased risk of arterial and venous thromboses. ${ }^{10}$ Patients with dysfibrinogenemia are often diagnosed after an incidental finding, for instance in routine hemostasis work-up before surgery. ${ }^{11}$ If the majority

(c) 2020 Georg Thieme Verlag KG Stuttgart - New York
DOI https://doi.org/ 10.1055/a-1182-3510. ISSN 0720-9355. 
Table 1 Classification of congenital fibrinogen disorders, adapted from Casini et al ${ }^{7}$

\begin{tabular}{|l|}
\hline Type and subtypes \\
\hline 1. Afibrinogenemia \\
\hline 1A. Patients with a bleeding phenotype or asymptomatic \\
\hline 1B. Patients with a thrombotic phenotype \\
\hline 2. Hypofibrinogenemia \\
\hline 2A. Severe: functional fibrinogen level $<0.5 \mathrm{~g} / \mathrm{L}$ \\
\hline 2B. Moderate: functional fibrinogen level between 0.5 and $0.9 \mathrm{~g} / \mathrm{L}$ \\
\hline 2C. Mild: functional fibrinogen level between $1 \mathrm{~g} / \mathrm{L}$ and lower limit of normal value \\
\hline 2D. Fibrinogen storage disease: familial hypofibrinogenemia with histologically proven accumulation of fibrin in hepatocytes \\
\hline 3. Dysfibrinogenemia \\
\hline 3A. Patients with bleeding or with thrombotic phenotype not fulfilling criteria for 3B \\
\hline 3B. Thrombotic-related dysfibrinogenemia: carriers of a thrombotic fibrinogen mutation ${ }^{\text {a }}$ or suffering from thrombotic events \\
with a first-degree familial thrombotic history (relatives with the same genotype) without any other thrombophilia \\
\hline 4. Hypodysfibrinogenemia \\
\hline 4A. Severe: antigenic fibrinogen level $<0.5 \mathrm{~g} / \mathrm{L}$ \\
\hline 4B. Moderate: antigenic fibrinogen level between 0.5 and $0.9 \mathrm{~g} / \mathrm{L}$ \\
\hline 4C. Mild: antigenic fibrinogen level between $1 \mathrm{~g} / \mathrm{L}$ and lower limit of normal value \\
\hline
\end{tabular}

aFibrinogen Dusart, Fibrinogen Caracas V, Fibrinogen ljmuiden, Fibrinogen New York 1, Fibrinogen Nijmegen, Fibrinogen Naples at homozygous state, Fibrinogen Melun.

of patients are asymptomatic or suffer from mild bleeding at diagnosis, the clinical course can sometimes be complicated by major bleeding (cumulative incidence estimated to 2.5 per 1,000 patient-years) and/or thromboses (cumulative estimated to 18.7 per 1,000 patient-years). ${ }^{12}$

In view of the heterogeneity of CFD, management of patients is challenging and an accurate diagnosis is mandatory. ${ }^{13}$ However, the segregation of the clinical phenotype is poor even in relatives with the same mutation. A more sophisticated approach is often needed to explain the relationship between fibrinogen genotype and phenotype. ${ }^{14}$ In this review, we summarize the fibrinogen work-up highlighting some common pitfalls and provide an update of the research on CFD.

\section{Laboratory Diagnosis of Congenital Fibrinogen Disorders}

As indicated in -Table 2, the initial fibrinogen work-up should include the measurement of the activated partial thromboplastin time (aPTT) and prothrombin time (PT) ${ }^{15}$ In afibrinogenemia, aPTT and PT are indefinitely prolonged

Table 2 Laboratory diagnosis of congenital fibrinogen disorders ${ }^{\mathrm{a}}$

\begin{tabular}{|c|c|c|c|c|c|}
\hline \multirow[t]{2}{*}{ Type } & \multirow[t]{2}{*}{ aPTT } & \multirow[t]{2}{*}{ PT } & \multicolumn{2}{|c|}{ Functional fibrinogen } & \multirow{2}{*}{$\begin{array}{l}\text { Antigen } \\
\text { fibrinogen }\end{array}$} \\
\hline & & & Clauss & PT-derived & \\
\hline Hypofibrinogenemia & $\begin{array}{l}\text { Normal or prolonged } \\
\text { according to the } \\
\text { fibrinogen level }\end{array}$ & $\begin{array}{l}\text { Normal or prolonged } \\
\text { according to the } \\
\text { fibrinogen level }\end{array}$ & Decreased & $\begin{array}{l}\text { Usually decreased } \\
\text { depending on the PT }\end{array}$ & Decreased \\
\hline Dysfibrinogenemia & $\begin{array}{l}\text { Normal or prolonged } \\
\text { according to the } \\
\text { fibrinogen level, the } \\
\text { method, and the } \\
\text { fibrinogen variant }\end{array}$ & $\begin{array}{l}\text { Normal or prolonged } \\
\text { according to the } \\
\text { fibrinogen level, the } \\
\text { method, and the } \\
\text { fibrinogen variant }\end{array}$ & $\begin{array}{l}\text { Usually } \\
\text { decreased }\end{array}$ & Usually normal ${ }^{b}$ & Normal \\
\hline Hypodysfibrinogenemia & $\begin{array}{l}\text { Normal or prolonged } \\
\text { according to the } \\
\text { fibrinogen level, the } \\
\text { method, and the } \\
\text { fibrinogen variant }\end{array}$ & $\begin{array}{l}\text { Normal or prolonged } \\
\text { according to the } \\
\text { fibrinogen level, the } \\
\text { method, and the } \\
\text { fibrinogen variant }\end{array}$ & Decreased & $\begin{array}{l}\text { Usually decreased } \\
\text { depending on the PT }\end{array}$ & Decreased \\
\hline
\end{tabular}

Abbreviations: aPTT, activated partial thromboplastin time; PT, prothrombin time.

${ }^{a}$ In addition to the proposed procedure, thrombin time can be performed as initial work of congenital fibrinogen disorder.

bThe PT-derived assay can be used as an alternative if immunoassays for fibrinogen antigen are unavailable. 
while in hypofibrinogenemia they are prolonged according to the fibrinogen level. ${ }^{13}$ In qualitative fibrinogen disorders they are variably prolonged. Shapiro et al showed in a study on 35 dysfibrinogenemic patients that the PT and aPTT are inconsistently normal or reduced, and dependent on the coagulometer and the activator chosen (further detailed below). ${ }^{16}$ Similar results have been reported in a recent exercise across 86 laboratories participating in the United Kingdom National External Quality Assessment Scheme for Blood Coagulation and the Prospective Rare Bleeding Disorders Database. According to the fibrinogen variant, the coefficients of variation of PT and aPTT were $71.8 \%$ and $40.8 \%$, respectively. ${ }^{17}$

As a second step, both activity and antigen of fibrinogen should be assessed. Fibrinogen activity is measured by the Clauss method or from the PT (i.e., PT-derived fibrinogen). Gravimetric assays such as clottable assays have long been considered as the gold standard to assess the fibrinogen concentration. However, they are time consuming and require a certain degree of expertise. A variety of immunological assays are available to assess the fibrinogen antigen. ${ }^{18}$ In afibrinogenemia fibrinogen levels will be undetectable while in hypofibrinogenemia a proportional decrease of both activity and antigen fibrinogen is observed. ${ }^{13}$ Dysfibrinogenemia is defined by the discrepancy between a normal antigen fibrinogen level and a decreased activity. ${ }^{19}$ The cut-off of 0.7 for the ratio Clauss/antigen is generally used for the screening of dysfibrinogenemia, ${ }^{20}$ even though it has never been properly validated. ${ }^{21}$ Hypodysfibrinogenemia is characterized by the discrepancy between decreased antigen and functional fibrinogen. ${ }^{22}$

Thrombin and batroxobin clotting time (reptilase time) are variably prolonged and provide additional information on the polymerization defects. ${ }^{23}$ In large series of dysfibrinogenemia $(n=101), 13.4$ and $11.3 \%$ had normal thrombin and reptilase time, respectively. ${ }^{12}$ When available, thrombin and reptilase time can complete the initial fibrinogen workup even though they are not mandatory for the diagnosis.

\section{Pitfalls in Diagnosis of Congenital Fibrinogen Disorders}

Diagnosis of afibrinogenemia is relatively easy by combining the clinical history (typically bleeding diathesis in childhood) with the characteristic infinitely prolonged coagulation assay. Nevertheless, distinguishing between afibrinogenemia and severe hypofibrinogenemia can be difficult due to the limited sensitivity of assays in case of fibrinogen levels $<0.5 \mathrm{~g} / \mathrm{L} .{ }^{24} \mathrm{This}$ distinction is clinically relevant as patients with severe hypofibrinogenemia are still able to secrete a small amount of fibrinogen in specific clinical settings such as pregnancy or trauma, compared with patients with afibrinogenemia. ${ }^{25} \mathrm{Sim}$ ilarly, based on coagulation assays it can be difficult to differentiate hypofibrinogenemia from hypodysfibrinogenemia. The ratio activity/antigen fibrinogen $<0.7$ has a sensitivity of approximately $85 \%$ to diagnosis of hypodysfibrinogenemia from hypofibrinogenemia. ${ }^{22}$ The difference between hypofibrinogenemia and hypodysfibrinogenemia is critical, since in the latter the fibrinogen variant is incorporated into the fibrin clot leading to an increased risk of bleeding and/or thrombosis. ${ }^{26}$ In such cases, mass spectrometry is useful to detect very small amount of fibrinogen and to determine whether the fibrinogen variant is secreted into the circulation. ${ }^{27}$

Diagnosis of dysfibrinogenemia is also complex. The fibrinogen activity can be either measured by the Clauss assay or derived from the change in light scatter or transmission during measurement of the PT time (PT-derived). ${ }^{18}$ The Clauss method gives markedly different results when various reagents are used. ${ }^{28}$ The wide variability of Clauss depends on the method of detection of fibrin clot formation (using optical or mechanical end-point detection systems). ${ }^{17}$ For some rare fibrinogen variants (e.g., Fibrinogen Longmont, Fibrinogen Bordeaux), fibrin polymerization is affected enough to modify the light scatter interpretation, while there is enough polymerization to increase plasma viscosity detected by an electromechanical analyzer. ${ }^{29}$ Several methods have been established for the assessment of the quantity of fibrinogen. If the heat precipitation method is considered the "gold standard," it is no more widely used due to technical difficulties. A variety of immunological assays provide an accurate estimation of the fibrinogen level. ${ }^{18}$

The PT-derived is not suitable for evaluation of the dysfibrinogenemia since it overestimates fibrinogen activity by a factor of 5 or $6 .^{30}$ On the contrary, the PT-derived method has a good correlation with the fibrinogen antigen $(r=0.923)$ and could be used as an alternative if immunoassays for fibrinogen antigen are not available. ${ }^{31}$ Discrepancy between PT-derived and Clauss suggests a dysfibrinogenemia. ${ }^{31}$ Recently, it has been reported that the clot wave form analysis could also be a good surrogate for the fibrinogen antigen. ${ }^{32}$ However, this innovative method needs to be validated in large cohorts before to be considered as a novel screening test.

\section{Genotype}

The genotype is mandatory to confirm the diagnosis and to identify types and subtypes of CFD. ${ }^{7}$ Studies aiming to identify causative mutations have relied on polymerized chain reaction amplification of coding regions and intron-exon junctions of the fibrinogen genes FGA, FGB, and FGG followed by Sanger sequencing. ${ }^{33}$ This approach is still valid, especially in the case of familial screening or in countries with limited resource, but Next Generation Sequencing technologies, in particular Whole Exome Sequencing, are becoming more readily accessible. It is anticipated that they will very soon become the method of choice. $^{34}$ To date, more than 250 causative mutations have been recorded in open-access databases. ${ }^{35}$ All afibrinogenemia mutations are null mutations, including two recurrent mutations ( $11 \mathrm{~kb}$ deletion of FGA and the splice site mutation c. $510+1 \mathrm{G}>\mathrm{T}$ of $F G A) .{ }^{36}$ Most of hypofibrinogenemia mutations are heterozygous and distributed across the three fibrinogen genes. ${ }^{37}$ Four hotspot mutations are prevalent in dysfibrinogenemia (c.103C $>\mathrm{T}$ and c.104G $>\mathrm{A}$ in FGA and c.901C $>$ T and c.902G $>\mathrm{A}$ in $F G G) .{ }^{38}$ Hypodysfibrinogenemia is often composite heterozygous with most of mutations in $F G G^{22}$ 
Some mutations are strongly correlated with clinical symptoms in hypofibrinogenemia and dysfibrinogenemia. A few mutations clustered in exon 8 and exon 9 of $F G G$ cause the hepatic storage disease (CFD subtype 2D according to the ISTH classification, - Table 1), characterized by a variable degree of histologically proven chronic liver disease and familial hypofibrinogenemia. ${ }^{39}$ Immunocytochemical analyses are necessary to confirm the diagnosis by revealing hepatocyte cytoplasmic inclusions immunoreactive to antifibrinogen antibodies. ${ }^{40}$ Several rare fibrinogen variants (e.g., Fibrinogen Dusart, Fibrinogen Caracas V or Nijmegen) confer a strong thrombotic risk (CFD subtype 3B according to the ISTH classification, -Table 1). ${ }^{41}$ Different mechanisms, often overlapping, participate to the increased risk of thrombosis in such dysfibrinogenemia. ${ }^{19}$ Failure of thrombin binding to fibrin leads to elevated levels of circulating thrombin. ${ }^{42}$ An abnormal structure of the fibrin affects the properties of the fibrin network. ${ }^{43}$ Impaired binding of plasminogen and tissue-type plasminogen activator to fibrin results in decreased fibrinolysis. ${ }^{44}$ It should be noted that all other fibrinogen variants can also contribute at different degrees to the overall patient's thrombotic phenotype, especially in the presence of coexisting inherited or acquired plasma hypercoagulability. ${ }^{41}$ In the case of identification of a new causative mutation, a proper experimental validation is necessary to evaluate the pathogenic role. ${ }^{45}$ The expression of the variant in transiently or stably transfected cells provides additional information and confirms, for instance, whether the fibrinogen mutant is fully assembled or secreted from the cell. ${ }^{46}$

\section{Research on Congenital Fibrinogen Disorders}

Specialized research laboratories perform additional functional, structural, and molecular investigations ( - Table 3 ). Besides the scientific interest, these could also potentially help to predict the clinical course for a given patient. ${ }^{19}$ One of the most peculiar characteristics of afibrinogenemia is the tendency to thrombosis. The underlying pathogenesis of thrombosis in patients with afibrinogenemia remains to be elucidated. ${ }^{47}$ Thrombin, which is not entrapped by the fibrin clot, ${ }^{48}$ is hence available for platelet activation and smooth muscle cell migration and proliferation, particularly in the arterial vessel wall. ${ }^{49}$ Subsampling studies (i.e., measurement of free thrombin in the fluid phase only) have shown an excess of thrombin in patients with afibrinogenemia, ${ }^{50}$ which can be normalized with fibrinogen substitution. ${ }^{51}$ The determination of a global hemostatic profile by thrombin generation assay in patients with afibrinogenemia could help to identify patients with increased thrombotic risk. ${ }^{52}$ Point-of-care viscoelastic tests such as the rotational thromboelastometry or the thromboelastography are used as surrogate endpoints in randomized trials on fibrinogen concentrates $^{53}$ or to guide the hemostatic management in high bleeding risk surgeries. ${ }^{54}$ However, they could have a limited sensitivity to certain changes in fibrin network structure and function, reducing their utility to a small number of patients. ${ }^{55}$ Further studies are needed to define whether these tests could be used to monitor the fibrinogen replacement ${ }^{56}$ or whether they could be of diagnosis or prognostic value. ${ }^{57,58}$

Table 3 Examples of functional, structural, and molecular investigations performed in research laboratories working on congenital fibrinogen disorders

\begin{tabular}{|l|l|}
\hline \multicolumn{2}{|l|}{ Fibrin polymerization and lysis } \\
\hline Turbidimetry & $\begin{array}{l}\text { Plotting light absorbance against time during clot formation; provide data on protofibril growth, } \\
\text { lateral fibril aggregation, internal fiber density, and fibrin lysis }\end{array}$ \\
\hline Thrombodynamics & Automated optical analyzer to continuously track spatial fibrin formation \\
\hline Fibrin network & Accurate visualization of the fibrin network and quantification of fibrin fiber diameter \\
\hline $\begin{array}{l}\text { Scanning electron } \\
\text { microscopy } \\
\text { Confocal microscopy }\end{array}$ & $\begin{array}{l}\text { Fibrin formation at the molecular level under physiological conditions allowing study of early fibrin } \\
\text { oligomer and protofibril formation }\end{array}$ \\
\hline Atomic force microscopy & $\begin{array}{l}\text { Measured by the Darcy constant (Ks), clot permeability gives an indication of the average pore size } \\
\text { between fibrin fibers }\end{array}$ \\
\hline Permeability & Measurement of clot rigidity and mechanical properties \\
\hline Viscoelastic properties & Basic information regarding the overall mechanical strength of the blood clot \\
\hline Rheometry & $\begin{array}{l}\text { The fibrin (with embedded paramagnetic bead) is imaged and displacement of the bead due to the } \\
\text { application of pulsating magnetic fields is tracked for the calculation of viscoelastic parameters }\end{array}$ \\
\hline Thromboelastography \\
\hline Magnetic tweezers
\end{tabular}


To better assess the phenotype of patients with qualitative fibrinogen disorders, an assessment of the properties of the fibrin clot can be performed. ${ }^{25}$ Accumulating evidence suggests that the fibrin clot plays a major role in the occurrence of thrombotic events and bleeding. ${ }^{59}$ The structure of the fibrin is a key determinant of the mechanical properties of the clot and of the resistance to fibrinolysis by the tissue plasminogen activator and the plasmin system. ${ }^{60}$ Among several research investigations, the polymerization and lysis assessment by turbidimetry, the measurement of permeability, and the evaluation of the fibrin clot structure by microscopy are often investigated in CFD. ${ }^{61}$ The turbidity curve is measured by plotting light absorbance with a selected wavelength against time during in vitro fibrin clot formation and lysis. ${ }^{62}$ The maximum absorbance directly relates to the fibrin-fiber cross-sectional area in a purified system and to the overall clot density in a plasma system. ${ }^{63}$ The clot lysis time is defined as the time from the midpoint of the ascending curve to the midpoint of the descending curve after the addition of a lytic activator. ${ }^{64}$ In a functional study on dysfibrinogenemia, all patients $(n=24)$ showed an abnormal fibrin polymerization compared with healthy controls. The clot lysis time was prolonged in patients with a thrombotic history while an increased permeability was statistically associated with a bleeding phenotype. ${ }^{65}$ The fibrin clot permeability is a measure of the porosity of the fibrin network reflecting the pore size between fibrin fibers. ${ }^{66}$ More permeable fibrin clots have larger pores and thicker fibrin fibers, which enhance the interaction between fibrin and plasmin leading to an increased fibrinolysis. ${ }^{14}$ Several imaging techniques are available to assess the fibrin clot structure, including scanning electron microscopy (SEM) and laser scanning confocal microscopy (LSCM). ${ }^{67}$ SEM gives a precise visualization and quantification of the fibrin-fiber diameter, even though with potential alteration due to fixation and drying procedures. LSCM obviates the need to add fixative chemicals but the fibrin-fiber diameters cannot reliably be measured. ${ }^{61}$ Such images can help to describe the fibrin clot network in patients with qualitative fibrinogen disorders. As an example, =Fig. 1 (A, B) provides an images of SEM in a patient with dysfibrinogenemia and a bleeding phenotype. Compared with the healthy control, the patient has a looser network composed of thicker fibers with larger pores. - Fig. 1 (C, D) shows a highly branched, tighter, and denser fibrin network in a patient carrier of the Fibrinogen Dusart with a thrombotic phenotype as compared with normal pool plasma. In the near future, more sophisticated imaging technologies including the thrombodynamics, ${ }^{68}$ or the high-resolution atomic force microscopy, or magnetic tweezers will enhance the knowledge of the structurefunction relations of fibrinogen variants. ${ }^{69}$ The evaluation of interplay between fibrinogen variants, coagulation
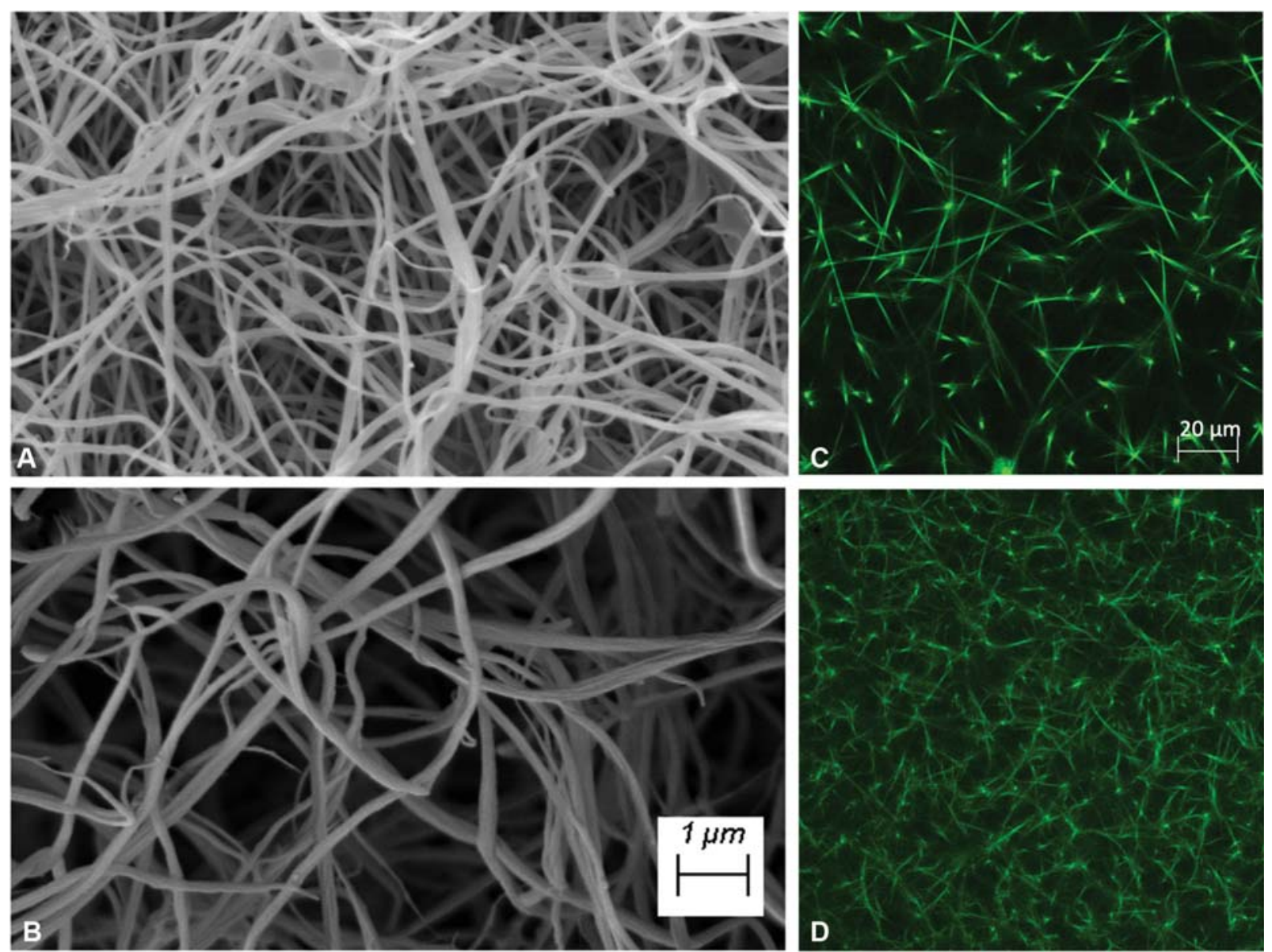

Fig. 1 Scanning electron microscopy in healthy control (A) and a patient with dysfibrinogenemia and a bleeding phenotype (B). Laser scanning confocal microscopy in healthy control (C) and a patient with dysfibrinogenemia and a thrombotic phenotype (D) (personal data). 
factors, blood cells, and endothelium will also provide a more global assessment of the determinants of the patient's clinical phenotype. ${ }^{60}$

\section{Conclusion}

An accurate diagnosis of CFDs is fundamental to optimize the patient's management. Translational research, from the evaluation of the molecular anomaly to the functional assessment of the biological phenotype, can eventually lead to a better estimation of the patient's hemostatic balance and finally to an individualized treatment.

\section{Conflict of Interest}

Dr. Casini reports personal fees from Octapharma, grants from CSL Behring, grants from NovoNordisk, nonfinancial support from Bayer, nonfinancial support from Sobi, outside the submitted work.

\section{References}

1 Pieters M, Wolberg AS. Fibrinogen and fibrin: an illustrated review. Res Pract Thromb Haemost 2019;3(02):161-172

2 Medved L, Weisel JW; Fibrinogen and Factor XIII Subcommittee of Scientific Standardization Committee of International Society on Thrombosis and Haemostasis. Recommendations for nomenclature on fibrinogen and fibrin. J Thromb Haemost 2009;7(02):355-359

3 Mosesson MW. Fibrinogen and fibrin structure and functions. J Thromb Haemost 2005;3(08):1894-1904

4 Menegatti M, Peyvandi F. Treatment of rare factor deficiencies other than hemophilia. Blood 2019;133(05):415-424

5 de Moerloose P, Neerman-Arbez M. Congenital fibrinogen disorders. Semin Thromb Hemost 2009;35(04):356-366

6 Paraboschi EM, Duga S, Asselta R. Fibrinogen as a pleiotropic protein causing human diseases: the mutational burden of $A \alpha, B \beta$, and $\gamma$ chains. Int J Mol Sci 2017;18(12):E2711

7 Casini A, Undas A, Palla R, Thachil J, de Moerloose P; Subcommittee on Factor XIII and Fibrinogen. Diagnosis and classification of congenital fibrinogen disorders: communication from the SSC of the ISTH. J Thromb Haemost 2018;16(09):1887-1890

8 Peyvandi F, Palla R, Menegatti M, et al; European Network of Rare Bleeding Disorders Group. Coagulation factor activity and clinical bleeding severity in rare bleeding disorders: results from the European Network of Rare Bleeding Disorders. J Thromb Haemost 2012;10(04):615-621

9 Stanciakova L, Kubisz P, Dobrotova M, Stasko J. Congenital afibrinogenemia: from etiopathogenesis to challenging clinical management. Expert Rev Hematol 2016;9(07):639-648

10 Nagler M, Kremer Hovinga JA, Alberio L, et al. Thromboembolism in patients with congenital afibrinogenaemia. Long-term observational data and systematic review. Thromb Haemost 2016;116 (04):722-732

11 Castaman G, Giacomelli SH, Biasoli C, Contino L, Radossi P. Risk of bleeding and thrombosis in inherited qualitative fibrinogen disorders. Eur J Haematol 2019;103(04):379-384

12 Casini A, Blondon M, Lebreton A, et al. Natural history of patients with congenital dysfibrinogenemia. Blood 2015;125(03):553-561

13 Neerman-Arbez M, de Moerloose P, Casini A. Laboratory and genetic investigation of mutations accounting for congenital fibrinogen disorders. Semin Thromb Hemost 2016;42(04):356-365

14 Undas A. How to assess fibrinogen levels and fibrin clot properties in clinical practice? Semin Thromb Hemost 2016;42(04):381-388

15 Verhovsek M, Moffat KA, Hayward CP. Laboratory testing for fibrinogen abnormalities. Am J Hematol 2008;83(12):928-931
16 Shapiro SE, Phillips E, Manning RA, et al. Clinical phenotype, laboratory features and genotype of 35 patients with heritable dysfibrinogenaemia. Br J Haematol 2013;160(02):220-227

17 Jennings I, Kitchen S, Menegatti M, et al. Potential misdiagnosis of dysfibrinogenaemia: data from multicentre studies amongst UK NEQAS and PRO-RBDD project laboratories. Int J Lab Hematol 2017;39(06):653-662

18 Mackie IJ, Kitchen S, Machin SJ, Lowe GD; Haemostasis and Thrombosis Task Force of the British Committee for Standards in Haematology. Guidelines on fibrinogen assays. $\mathrm{Br} \mathrm{J}$ Haematol 2003;121(03):396-404

19 Casini A, Neerman-Arbez M, Ariëns RA, de Moerloose P. Dysfibrinogenemia: from molecular anomalies to clinical manifestations and management. J Thromb Haemost 2015;13(06):909-919

20 Peyvandi F. Epidemiology and treatment of congenital fibrinogen deficiency. Thromb Res 2012;130(Suppl 2):S7-S11

21 Krammer B, Anders O, Nagel HR, Burstein C, Steiner M. Screening of dysfibrinogenaemia using the fibrinogen function versus antigen concentration ratio. Thromb Res 1994;76(06):577-579

22 Casini A, Brungs T, Lavenu-Bombled C, Vilar R, Neerman-Arbez M, de Moerloose P. Genetics, diagnosis and clinical features of congenital hypodysfibrinogenemia: a systematic literature review and report of a novel mutation. J Thromb Haemost 2017;15 (05):876-888

23 Chandler JB, Siddon AJ, Bahel P, Torres R, Rinder HM, Tormey CA. Modified approach to fibrinogen replacement in the setting of dysfibrinogenaemia. J Clin Pathol 2019;72(02):177-180

24 Asselta R, Duga S, Spena S, et al. Missense or splicing mutation? The case of a fibrinogen Bbeta-chain mutation causing severe hypofibrinogenemia. Blood 2004;103(08):3051-3054

25 Casini A, de Moerloose P. Can the phenotype of inherited fibrinogen disorders be predicted? Haemophilia 2016;22(05):667-675

26 Ridgway HJ, Brennan SO, Faed JM, George PM. Fibrinogen Otago: a major alpha chain truncation associated with severe hypofibrinogenaemia and recurrent miscarriage. Br J Haematol 1997;98 (03):632-639

27 Brennan SO, Mangos H, Faed JM. Benign FGB (148Lys $\rightarrow$ Asn, and 448Arg $\rightarrow$ Lys), and novel causative 2 $211 \mathrm{Tyr} \rightarrow$ His mutation distinguished by time of flight mass spectrometry in a family with hypofibrinogenaemia. Thromb Haemost 2014;111(04):679-684

28 Vasse M, Francois D, Van Dreden P, de Mazancourt P. Different sensitivity of von Clauss reagents for the diagnosis of dysfibrinogenemia. Eur J Haematol 2020;104(01):70-71

29 Lefkowitz JB, DeBoom T, Weller A, Clarke S, Lavrinets D. Fibrinogen Longmont: a dysfibrinogenemia that causes prolonged clotbased test results only when using an optical detection method. Am J Hematol 2000;63(03):149-155

30 Miesbach W, Schenk J, Alesci S, Lindhoff-Last E. Comparison of the fibrinogen Clauss assay and the fibrinogen PT derived method in patients with dysfibrinogenemia. Thromb Res 2010;126(06): e428-e433

31 Xiang L, Luo M, Yan J, et al. Combined use of Clauss and prothrombin time-derived methods for determining fibrinogen concentrations: screening for congenital dysfibrinogenemia. J Clin Lab Anal 2018;32(04):e22322

32 Suzuki A, Suzuki N, Kanematsu T, et al. Clot waveform analysis in Clauss fibrinogen assay contributes to classification of fibrinogen disorders. Thromb Res 2019;174:98-103

33 Casini A, Blondon M, Tintillier V, et al. Mutational epidemiology of congenital fibrinogen disorders. Thromb Haemost 2018;118(11): 1867-1874

34 Moret A, Zúñiga Á, Ibáñez M, et al. Clinical and molecular characterization by next generation sequencing of Spanish patients affected by congenital deficiencies of fibrinogen. Thromb Res 2019;180:115-117

35 Hanss M, Biot F. A database for human fibrinogen variants. Ann N Y Acad Sci 2001;936:89-90 
36 Neerman-Arbez M, de Moerloose P. Mutations in the fibrinogen gene cluster accounting for congenital afibrinogenemia: an update and report of 10 novel mutations. Hum Mutat 2007;28(06): 540-553

37 Asselta R, Duga S, Tenchini ML. The molecular basis of quantitative fibrinogen disorders. J Thromb Haemost 2006;4(10):2115-2129

38 Neerman-Arbez M, Casini A. Clinical consequences and molecular bases of low fibrinogen levels. Int J Mol Sci 2018;19(01):E192

39 Callea F, Giovannoni I, Sari S, et al. Fibrinogen gamma chain mutations provoke fibrinogen and apolipoprotein B plasma deficiency and liver storage. Int J Mol Sci 2017;18(12):E2717

40 Asselta R, Robusto M, Braidotti P, et al. Hepatic fibrinogen storage disease: identification of two novel mutations (p.Asp316Asn, fibrinogen Pisa and p.Gly366Ser, fibrinogen Beograd) impacting on the fibrinogen $\gamma$-module. J Thromb Haemost 2015;13(08): 1459-1467

41 Haverkate F, Samama M. Familial dysfibrinogenemia and thrombophilia. Report on a study of the SSC Subcommittee on Fibrinogen. Thromb Haemost 1995;73(01):151-161

42 Meh DA, Mosesson MW, Siebenlist KR, et al. Fibrinogen naples I (B beta A68T) nonsubstrate thrombin-binding capacities. Thromb Res 2001;103(01):63-73

43 Weisel JW, Litvinov RI. Mechanisms of fibrin polymerization and clinical implications. Blood 2013;121(10):1712-1719

44 Collet JP, Soria J, Mirshahi M, et al. Dusart syndrome: a new concept of the relationship between fibrin clot architecture and fibrin clot degradability: hypofibrinolysis related to an abnormal clot structure. Blood 1993;82(08):2462-2469

45 Smith N, Bornikova L, Noetzli L, et al. Identification and characterization of novel mutations implicated in congenital fibrinogen disorders. Res Pract Thromb Haemost 2018;2(04):800-811

46 Asselta R, Platè M, Robusto M, et al. Clinical and molecular characterisation of 21 patients affected by quantitative fibrinogen deficiency. Thromb Haemost 2015;113(03):567-576

47 Korte W, Poon MC, Iorio A, Makris M. Thrombosis in inherited fibrinogen disorders. Transfus Med Hemother 2017;44(02): 70-76

48 Mosesson MW. Update on antithrombin I (fibrin). Thromb Haemost 2007;98(01):105-108

49 Santoro C, Massaro F, Venosi S, et al. Severe thrombotic complications in congenital afibrinogenemia: a pathophysiological and management dilemma. Semin Thromb Hemost 2016;42(05): 577-582

50 Kumar R, Béguin S, Hemker HC. The influence of fibrinogen and fibrin on thrombin generation-evidence for feedback activation of the clotting system by clot bound thrombin. Thromb Haemost 1994;72(05):713-721

51 Korte W, Feldges A. Increased prothrombin activation in a patient with congenital afibrinogenemia is reversible by fibrinogen substitution. Clin Investig 1994;72(05):396-398

52 Young GA, Carmona R, Cano Garcia V. Thromboelastography and thrombin generation assay in inherited afibrinogenemia. Haemophilia 2018;24(06):e410-e416

53 Ross C, Rangarajan S, Karimi M, et al. Pharmacokinetics, clot strength and safety of a new fibrinogen concentrate: randomized comparison with active control in congenital fibrinogen deficiency. J Thromb Haemost 2018;16(02):253-261

54 Simurda T, Casini A, Stasko J, et al. Perioperative management of a severe congenital hypofibrinogenemia with thrombotic phenotype. Thromb Res 2020;188:1-4
55 Galanakis DK, Neerman-Arbez M, Brennan S, et al. Thromboelastographic phenotypes of fibrinogen and its variants: clinical and non-clinical implications. Thromb Res 2014;133(06): $1115-1123$

56 Kalina U, Stöhr HA, Bickhard H, et al. Rotational thromboelastography for monitoring of fibrinogen concentrate therapy in fibrinogen deficiency. Blood Coagul Fibrinolysis 2008;19(08):777-783

57 Treliński J, Pachniewska K, Matczak J, Robak M, Chojnowski K. Assessment of selected ROTEM parameters, kinetics of fibrinogen polymerization and plasmin amidolytic activity in patients with congenital fibrinogen defects. Adv Clin Exp Med 2016;25(06): 1255-1263

58 Zhou J, Xin Y, Ding Q et al. Thromboelastography predicts risks of obstetric complication occurrence in (hypo)dysfibrinogenemia patients under non-pregnant state. Clin Exp Pharmacol Physiol 2016;43(02):149-156

59 Bridge KI, Philippou H, Ariëns R. Clot properties and cardiovascular disease. Thromb Haemost 2014;112(05):901-908

60 Kattula S, Byrnes JR, Wolberg AS. Fibrinogen and fibrin in hemostasis and thrombosis. Arterioscler Thromb Vasc Biol 2017;37 (03):e13-e21

61 Mihalko E, Brown AC. Clot structure and implications for bleeding and thrombosis. Semin Thromb Hemost 2020;46(01):96-104

62 Pieters M, Philippou H, Undas A, de Lange Z, Rijken DC, Mutch NJ; Subcommittee on Factor XIII and Fibrinogen, and the Subcommittee on Fibrinolysis. An international study on the feasibility of a standardized combined plasma clot turbidity and lysis assay: communication from the SSC of the ISTH. J Thromb Haemost 2018;16(05):1007-1012

63 Pieters M, Guthold M, Nunes CM, de Lange Z. Interpretation and validation of maximum absorbance data obtained from turbidimetry analysis of plasma clots. Thromb Haemost 2020;120(01): 44-54

64 Longstaff C. Measuring fibrinolysis: from research to routine diagnostic assays. J Thromb Haemost 2018;16(04):652-662

65 Casini A, Duval C, Pan X, Tintillier V, Biron-Andreani C, Ariëns RAS. Fibrin clot structure in patients with congenital dysfibrinogenaemia. Thromb Res 2016;137:189-195

66 Pieters M, Undas A, Marchi R, De Maat MP, Weisel J, Ariëns RA; Factor XIII And Fibrinogen Subcommittee Of The Scientific Standardisation Committee Of The International Society For Thrombosis And Haemostasis. An international study on the standardization of fibrin clot permeability measurement: methodological considerations and implications for healthy control values. J Thromb Haemost 2012;10(10):2179-2181

67 Ariëns RA. Fibrin(ogen) and thrombotic disease. J Thromb Haemost 2013;11(Suppl 1):294-305

68 Panteleev MA, Ovanesov MV, Kireev DA, et al. Spatial propagation and localization of blood coagulation are regulated by intrinsic and protein C pathways, respectively. Biophys J 2006;90(05): 1489-1500

69 Domingues MM, Macrae FL, Duval C, et al. Thrombin and fibrinogen $\gamma^{\prime}$ impact clot structure by marked effects on intrafibrillar structure and protofibril packing. Blood 2016;127(04):487-495

70 Pieters M, Guthold M, Nunes CM, de Lange Z. Interpretation and validation of maximum absorbance data obtained from turbidimetry analysis of plasma clots. Thromb Haemost 2020;120(01):44-54

71 Tutwiler V, Litvinov RI, Lozhkin AP, et al. Kinetics and mechanics of clot contraction are governed by the molecular and cellular composition of the blood. Blood 2016;127(01):149-159 